\title{
The Unconscious Affection Factor: Exploring the Dual Facets of Customer-Perceived Value and Their Impact on Brand Attachment: An Abstract
}

\author{
Sascha Langner, Steffen Schmidt, Levke Albertsen, \\ Evmorfia Karampournioti, and Klaus-Peter Wiedmann
}

\begin{abstract}
The customer-perceived value (CPV) has been identified as one of the most important factors considering customer's decision-making. The reason why people prefer specific brands over others is directly related to the perceived (and experienced) values of the considered brand. In the past, most research with regard to brand-related marketing issues relies on conventional self-reporting scales (e.g., Esch et al., 2008). However, an impressive number of studies in the field of cognitive psychology and neuroscience indicate that most mental processes in the human's brain are processed unconsciously without any conscious (explicit) awareness (e.g., Nisbett \& Wilson, 1977; Weber et al., 2009). That introspective inaccessibility limits consumers to fully articulate their feelings and thoughts about a brand. Specifically, conventional research of customer-perceived value often falls back on conventional measures like self-reports which are able to capture judgments and opinions on an explicit level, but not on an implicit (un-subconscious/subconscious) level (e.g., Smith \& Colgate, 2007). Against that backdrop, a substantial understanding of brand-related customer-perceived value implies an advanced comprehension not only of conscious, so-called explicit processes, but also of the unconscious, so-labeled implicit processes. In this respect, the present exploratory work introduces a multifaceted modeling of customer-perceived value incorporating explicit and implicit processes in a combined measurement approach and their impact on brand attachment. The findings of the present exploratory study show that brand attachment is strongly driven by implicit and explicit facets of CPV.
\end{abstract}

References Available Upon Request

\footnotetext{
S. Langner $\bullet$ S. Schmidt $(\varangle) \bullet$ L. Albertsen $\bullet$ E. Karampournioti $\bullet$ K.-P. Wiedmann

Leibniz University of Hanover, Hanover, Germany

e-mail: langner@m2.uni-hannover.de; schmidt@m2.uni-hannover.de;

albertsen@m2.uni-hannover.de; karampournioti@m2.uni-hannover.de;

wiedmann@m2.uni-hannover.de
} 